\title{
Safety Map: A Unified Representation for Biomechanics Impact Data and Robot Instantaneous Dynamic Properties
}

\author{
Nico Mansfeld ${ }^{1}$, Mazin Hamad ${ }^{2}$, Marvin Becker ${ }^{2}$, Antonio Gonzales Marin ${ }^{1}$ and Sami Haddadin ${ }^{2}$
}

\begin{abstract}
Close physical human-robot interaction makes it essential to ensure human safety. In particular, the intrinsic safety characteristics of a robot in terms of potential human injury have to be understood well. Then, minimal potential harm can be made a key requirement already at an early stage of the robot design. In this paper, we propose the safety map concept, a map that captures human injury occurrence and robot inherent global or task-dependent safety properties in a unified manner, making it a novel, powerful, and convenient tool to quantitatively analyze the safety performance of a certain robot design. In this paper, we derive the concept and elaborate the map representations of the PUMA 560, KUKA Lightweight Robot IV+, and injury data of the human head and chest. For the latter, we classify and summarize the most relevant impact studies and extend existing literature overviews. Finally, we validate our approach by deriving the safety map for a pick and place task, which allows to assess human safety and guide the task/robot designer how to take measures in order to account for both safety and task performance requirements, respectively.
\end{abstract}

Index Terms-Robot Safety, Physical Human-Robot Interaction, Human-Centered Robotics

\section{INTRODUCTION}

$\mathbf{E}$ NSURING human safety is a primary concern in physical human-robot interaction (pHRI), as physical contact is part of the process and potentially dangerous collisions may occur. The investigation of injury mechanisms and the development of safe mechanical designs and control strategies are still ongoing research topics and many efforts have been taken until now.

For ensuring collision safety in terms of kinematics and mechanics, lightweight manipulator design is essential. In addition to lightweight but rather rigid manipulators, intrinsic joint elasticity and soft covering were recently employed to improve collision safety [1]-[3]. The benefit of joint elasticity on collision safety, however, has to be treated more differentiated [4]. For most robots, the selection of inertial and

Manuscript received: September, 10, 2017; Revised December, 12, 2017; Accepted January, 14, 2018.

This paper was recommended for publication by Editor Paolo Rocco upon evaluation of the Associate Editor and Reviewers' comments. This work was supported by the European Union's Horizon 2020 research and innovation programme as part of the projects ILIAD and SoftPro under grant no. 732737 and 688857, by the European Commission's Seventh Framework Programme as part of the project EuRoC under grant no. 608849, and by the Alfried Krupp von Bohlen und Halbach Foundation. We would like to thank Gauthier Hentz for his support during the collection and classification of biomechanics collision data.

${ }^{1}$ Nico Mansfeld and Antonio Gonzales Marin are with the Institute of Robotics and Mechatronics, German Aerospace Center (DLR), Wessling, Germany, nico.mansfeldedlr.de

${ }^{2}$ Mazin Hamad, Marvin Becker, and Sami Haddadin are with the Institute of Automatic Control, Leibniz Universität Hannover (LUH), Germany, sami.haddadineirt.uni-hannover. de

Digital Object Identifier (DOI): see top of this page.

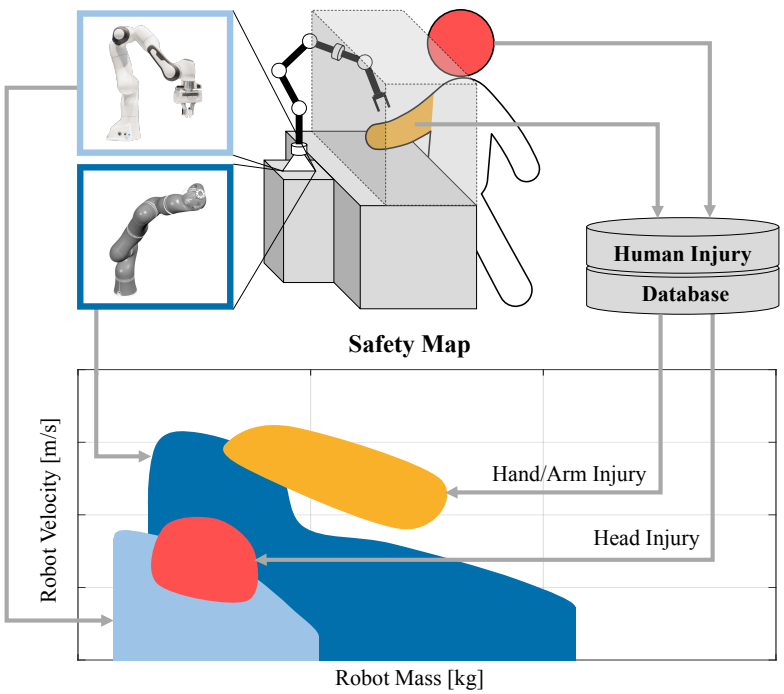

Fig. 1. Safety map concept. The global or task-specific (gray interaction area) mass/velocity ranges of different robots (here: DLR Lightweight Robot and Franka Emika Panda) and injury occurrence of human body parts are represented in a unified manner in the safety map. The injury data associated with the considered body parts is obtained from an injury database.

elastic properties is usually driven by certain design decisions. In contrast, the authors of [5], [6] proposed to integrate quantitative safety (and performance) criteria already in the mechanical design phase.

In terms of safe control, many metrics- and model-based approaches were proposed [6]-[10]. A major well-known drawback of model- and metrics-based ratings of a robot's safety characteristics is that the consistency with medically observed injury is often insufficient. This was pointed out in our previous work [11], where we proposed to directly associate instantaneous robot collision behavior, i. e., reflected mass, velocity, and contact geometry to observed human injury for a realistic and a-priori model-independent safety analysis. In contrast to other approaches no intermediate physical quantities such as force or pressure had to be associated with injury (however, could be). Then, so-called safety curves can be derived that provide a maximum biomechanically safe velocity as a function of instantaneous inertial robot properties. These representations were further developed into the safe velocity controller Safe Motion Unit that limits the instantaneous robot speed by respecting the safety curves, therefore ensuring human safety even in case of entirely unforeseen collisions.

In this paper, we further employ this idea to deduce a global perspective of a robot's collision safety. More specifically, we propose the concept of a safety map, which reflects both the 
robot global dynamic properties for a desired granularity and the relationship between collision input parameters and human injury. The safety map enables the user to address following (among other) questions:

- Is the considered robot capable of producing a certain type of injury during unforeseen collisions in my application?

- Where are the most dangerous areas in the reachable robot workspace?

- How do the robot safety characteristics compare with other performance indices? For example, how dangerous is the robot in its most dexterous workspace?

- How does the robot compare to other robots in terms of safety characteristics?

For deriving the safety map representation and relating entire robot designs to available biomechanics safety data, we analyze the reflected mass and maximum velocity of a robot in task-dependent workspace sets. This is done for two exemplary robots, namely the PUMA 560 and the KUKA Lightweight Robot IV+ (LWR). Regarding injury data, we extend our initial injury data literature overview [12] by a thorough summary on the human head and chest. We classify, validate, and process a significant amount of relevant data from 50 years of biomechanics injury research into the mass/velocity representation and link it to the proposed safety maps.

In summary, the safety map concept may serve as a global safety assessment framework for entire robot designs without the need of simplifications. This makes it a valuable tool not only for safety-oriented planning and control but in particular for safer robot design.

The remainder of this paper is organized as follows. In Section II, we describe the concept of safety maps in more detail. In Section III, we briefly describe the collision model that is used to classify and compare collision experiments. The results of our literature review on injury data for the human head and chest are provided in Section IV. In Section V, we describe how robot kinematic and dynamic parameters can be processed towards the safety map representation and provide examples for the PUMA 560 and the $L W R I V+$. Section VI addresses how the safety map can be utilized to evaluate safety in practical applications. Finally, Section VII concludes the paper.

\section{Definition SAFETy MAP}

In previous works, trajectories or "representative" configurations were related to human injury probability or safety metrics in order to locally avoid unwanted injury via planning or control. In this paper, we propose to

- relate entire robot designs, i.e., the mass/velocity pairs for the reachable workspace, respectively a taskdependent subset, to

- human injury data, which may

- originate from different types of experiments and disciplines (robotics, forensics, biomechanics, simulations etc.),

- consider different body parts,

- impactor curvatures (blunt, edgy, sharp), and

- collision cases (constrained, unconstrained),

- in the same "coordinate system", namely the plane spanned by the robot reflected mass and velocity.

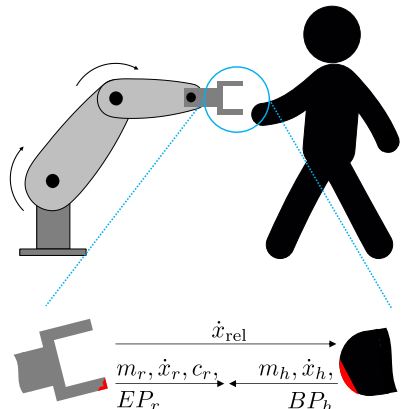

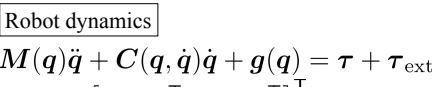
$\boldsymbol{J}(\boldsymbol{q})=\left[\boldsymbol{J}_{v}(\boldsymbol{q})^{\top}, \boldsymbol{J}_{w}(\boldsymbol{q})^{\top}\right]^{\top}$

Reflected mass

$\boldsymbol{\Lambda}(\boldsymbol{q})=\left(\boldsymbol{J}(\boldsymbol{q}) \boldsymbol{M}(\boldsymbol{q})^{-1} \boldsymbol{J}(\boldsymbol{q})^{\top}\right)^{-1}$

$\Rightarrow m_{r}=m_{u}(\boldsymbol{q})=\left[\boldsymbol{u}^{\top} \boldsymbol{\Lambda}_{v}(\boldsymbol{q})^{-1} \boldsymbol{u}\right]^{-1}$

Cartesian velocity

$\dot{\boldsymbol{x}}=\boldsymbol{J}_{v}(\boldsymbol{q}) \dot{\boldsymbol{q}}$

$\Rightarrow \dot{x}_{r}=\dot{x}_{u}(\boldsymbol{q})=\boldsymbol{u}^{\top} \dot{\boldsymbol{x}}(\boldsymbol{q})$

Fig. 2. Collision model for representing the instantaneous dynamic properties of the impactor/robot and subject/human. In the robot dynamic equations, $\boldsymbol{q} \in \mathbb{R}^{n}$ denotes the generalized coordinates, $\boldsymbol{M}(\boldsymbol{q}) \in \mathbb{R}^{n \times n}$ the symmetric, positive definite mass matrix, $\boldsymbol{C}(\boldsymbol{q}, \dot{\boldsymbol{q}}) \in \mathbb{R}^{n \times n}$ the Coriolis and centrifugal matrix, and $\boldsymbol{g}(\boldsymbol{q}) \in \mathbb{R}^{n}$ the gravity torque vector. The motor joint torque is denoted $\tau \in \mathbb{R}^{n}$ and the external joint torques $\tau_{\text {ext }} \in \mathbb{R}^{n}$. The Jacobian matrix associated with the impact location is $\boldsymbol{J}(\boldsymbol{q}) \in \mathbb{R}^{6 \times n}$ and the Cartesian mass matrix is $\boldsymbol{\Lambda}(\boldsymbol{q}) \in \mathbb{R}^{6 \times 6}$. The scalar mass and velocity in normalized Cartesian direction $\boldsymbol{u} \in \mathbb{R}^{3}$ are denoted $m_{u}(\boldsymbol{q}) \in \mathbb{R}$ and $\dot{x}_{u}(\boldsymbol{q}) \in \mathbb{R}$.

This global representation is denoted safety map, the concept is illustrated in Fig. 1.

The representations of human injury data and robot dynamic properties shall be independent from each other, meaning there is no direct dependency of injury data on robot data and vice versa. This allows to compare different robot designs with the same injury data. Ideally, the injury representation is available in a purely data-driven functional relation, linking robot input parameters to human injury or pain directly. However, also injury criteria based on physical quantities can be illustrated in the mass/velocity plane, if a model or functional relation is available that provides the desired mapping.

By aggregating the representations of human injury data and dynamic robot properties, the safety map enables a robot/task designer to assess the considered robot(s) in combination with the task specification in terms of safety already at a very early planning stage. For example, in Fig. 1 the mass/velocity ranges of the two exemplary robots intersect with the head injury data, which means that both robots may harm the human head during unforeseen collisions. Hand/arm injury, however, may only be produced by the second robot $^{1}$.

In Sec. VI, we describe in more detail how the safety map can be integrated as an evaluation tool into safety assessment and safe robot/task design. In the following sections III - V, we address how injury data and robot dynamic properties can be processed towards the safety map representation systematically.

\section{COLlision Model}

In this section, we describe the model that serves to determine the robot and human instantaneous collision dynamics and parameters, see Fig. 2. The model follows our approach taken in [11] and is based on the idea that any mechanical system (here: impactor/robot and subject/human) can be represented by an instantaneous scalar mass, velocity, and surface properties in a certain Cartesian direction. For more information on each parameter, please refer to the cited literature.

\footnotetext{
${ }^{1}$ Please note that these are no general conclusions as for illustrative reasons the data in Fig. 1 is fictitious.
} 
Impactor/Robot Parameters: The impactor/robot is modeled in terms of its instantaneous mass $m_{r}$, velocity $\dot{x}_{r}$, curvature $c_{r}$, and elastic surface properties $E P_{r}$.

In biomechanics drop or pendulum tests a scalar mass can typically be associated with the impactor due to its minimalistic design [13], [14]. For robots, the so-called reflected mass in a certain Cartesian direction represents the mass perceived during a collision [15]. The essential equations for calculating the Cartesian reflected mass and velocity are provided in Fig. 2 (right). The impactor surface may either be blunt, edgy, or sharp. In most publications, general geometric shapes such as cylinders or flat circular plates are used, which were designed such that precise impacts can be delivered to a desired subject location. In [11], [12] principal geometric primitives (sphere, edge, etc.) were identified and clustered. With these primitives it is possible to classify most impactors used in biomechanics and robotics impact experiments.

Subject/Human Impact Parameters: The subject is represented in terms of the impact location $B P_{h}$, instantaneous mass $m_{h}$, and velocity $\dot{x}_{h}$.

The impact location of the human is a characteristic landmark of the musculoskeletal system such as the frontal bone or maxilla in the human head [16]. We assume that the impactor's Cartesian direction of motion coincides with the surface normal of the respective body part. This agrees with the experimental design in almost all biomechanics and robotics publications. We denote the relative velocity between subject and impactor ${ }^{2}$ as $\dot{x}_{\text {rel }}=\left|\dot{x}_{r}-\dot{x}_{h}\right|$.

To estimate the human effective mass at the contact location (if not reported), one can a) use a model of the human body based on the geometrical and inertial properties [17], [18], or b) fit the parameters of a mathematical collision model, e. g., a fully (in)elastic impact in a mass-spring-mass system, by conducting suitable impact experiments.

\section{Synopsis of Human Head \& Chest Impact Data}

We provide a summary of most relevant biomechanics and robotics collision experiments on the human head and chest in Tab. I. This summary is a result of an extensive literature study and extends our initial literature survey reported in [12]. For our data-driven approach of relating collision input parameters to resulting injury, the collected data is of high value because it allows to compare the results from different experiments, determine whether a certain robot may produce injury, verify mathematical collision models, etc. Most of the available biomechanical literature stems from automotive injury analysis with the focus on more severe injuries. The head and chest are usually of particular interest, which is why much collision data has been generated for these body parts.

In Tab. I we use the previously described collision model to classify and quantify all relevant parameters. Furthermore, we distinguish between different subject types, collision scenarios, and experimental setups. In terms of the collision scenario, we distinguish between impacts, where the subject is unconstrained, constrained, or partially constrained [19]. The latter is characterized only by a part of the subject being clamped, which is not directly in contact with the impactor. In Tab. I we use following abbreviations: U: unconstrained, C: constrained, PC: partially constrained. From the biomechanics experiments,

${ }^{2}$ We only consider robot and human velocities that result in a collision. we identify four principal setups. In setup I and II, the freefall principle is used, where I) the impactor or II) the subject is accelerated. In setup III and IV, the impact is delivered horizontally, where either III) the subject or IV) the impactor is at rest. For each setup, different human collision scenarios are possible, i. e., the respective body part can be constrained, unconstrained, or partially constrained.

The selected classification allows us to store and process the experimental data in a systematic fashion using a database. In Tab. I, we summarize the experimental conditions for each impact series. Every impact with its exact parameters and injury evaluation has a separate entry in the database. The graphical representation of the relationship between impact parameters and injury severity is also illustrated in Fig. 3 for a selection of the listed experiments.

The collection of biomechanics injury data is an ongoing process. In this paper, we provide relevant results for the mentioned body parts, a thorough overview for all human body parts and a detailed description of the database is subject to future work.

a) Head Impact Data: In the upper half of Tab. I we provide an overview of data from facial and cranial bone injury analysis. In Fig. 3 (left) we illustrate the results of experiments on the frontal bone, where a flat impactor is accelerated towards the subject (setups I and III) [20], [24], [26]. We relate the collision input parameters mass and velocity to injury severity, which is the occurrence of skull fracture or subfractures (e.g., hairline cracks) in this example.

b) Chest Impact Data: A significant amount of experiments on chest injury analysis was conducted in [30]-[38]. In order to better understand thoracic trauma in frontal impacts, a more recent extensive crash-test program was established [39]. In robotics, series of chest crash-test experiments were conducted in [22], [40], where several lightweight and heavyduty robots were used. Both dynamic unconstrained (with $K U K A K R 6$ and KR500) and quasistatic constrained (with $L W R$ III) frontal chest impacts were carried out. An overview of the most relevant impact experiments for the chest in both biomechanics and robotics is provided in the lower half of Tab. I. In Fig. 3 (right), we illustrate the relationship between collision input parameters and injury severity for selected chest impact experiments.

\section{Deriving Global Robot Dynamic Properties FOR SAFETY MAP REPRESENTATION}

Having collected, classified, and processed human injury data, we now describe how the kinematic and dynamic characteristics of a robot can be mapped to a mass/velocity range in the safety map in order to represent the robot properties on a global or local, task-dependent, scale. We seek to determine the reflected mass and maximum velocity for all reachable poses, i.e., Cartesian positions and orientations, and in every Cartesian direction $\boldsymbol{u}$. One main idea of the concept is to calculate the global dynamic properties of a robot design for a desired granularity only once. Afterwards, the data associated with task-dependent subsets of the robot workspace can be extracted, certain trajectories or single static configurations can also be analyzed by interpolating the data, thus allowing for different degrees of granularity in the safety analysis.

The procedure for computing the global robot dynamic properties consists of four steps, namely 
TABLE I

OVERVIEW OF SELECTED IMPACT EXPERIMENTS FROM BIOMECHANICS AND ROBOTICS LITERATURE FOR THE HUMAN HEAD AND CHEST.

\begin{tabular}{|c|c|c|c|c|c|c|c|c|}
\hline \multirow{2}{*}{ Body Part } & \multirow{2}{*}{ Exp. Setup } & \multirow{2}{*}{ Case } & \multicolumn{2}{|r|}{ Impactor } & \multirow{2}{*}{ Subject } & \multirow{2}{*}{ Mass [kg] } & \multirow{2}{*}{ Velocity $[\mathrm{m} / \mathrm{s}]$} & \multirow{2}{*}{ Ref. } \\
\hline & & & Primitive & Parameters & & & & \\
\hline Frontal & III & $\mathrm{PC}$ & Flat circular & $35 \mathrm{~mm}$ radius & Cadaver & $28.9-48.3$ & $3.39-6.99$ & {$[20]$} \\
\hline Frontal & II & $\mathrm{U}$ & Edge & $10 \mathrm{~mm}$ radius & Cadaver & 14.5 & $3.0-4.2$ & [14] \\
\hline Frontal & I & $\mathrm{U}$ & Edge & $25.4 \mathrm{~mm} \& 7.9 \mathrm{~mm}$ radius & Cadaver & 4.54 & $1.44-3.22$ & [13] \\
\hline Frontal, Occipital & II & $\mathrm{U}$ & Flat rectangular & Padded, $120 \mathrm{~mm} \times 80 \mathrm{~mm}$ & Cadaver & 5 & $2.8-7.0$ & [21] \\
\hline Frontal & III & $\mathrm{U}, \mathrm{PC}$ & Sphere & Rigid, $120 \mathrm{~mm}$ radius & HIII Dummy & $4.0,67.0,1980.0$ & $0.2-4.2$ & {$[22]$} \\
\hline Frontal & II & $\mathrm{PC}$ & Sphere & Padded, $76.2 \mathrm{~mm}-203.2 \mathrm{~mm}$ radius & Cadaver & $4.54-6.49$ & $2.95-3.54$ & {$[23]$} \\
\hline Frontal, Temporo-Parietal & II & PC & Edge & Padded, $3.2 \mathrm{~mm}-25.4 \mathrm{~mm}$ radius & Cadaver & $3.18-6.49$ & $2.2-4.37$ & {$[23]$} \\
\hline $\begin{array}{l}\text { Frontal, Temporo-Parietal, } \\
\text { Occipital }\end{array}$ & II & PC & Flat & Padded & Cadaver & $3.31-5.9$ & $2.23-5.43$ & {$[23]$} \\
\hline $\begin{array}{l}\text { Frontal, Zygoma, Mandible, } \\
\text { Maxilla }\end{array}$ & III & $\mathrm{U}, \mathrm{PC}$ & Flat circular & $\begin{array}{l}\text { Padded, } \\
14.3 \mathrm{~mm} \& 32.7 \mathrm{~mm} \text { radius }\end{array}$ & Cadaver & $0.9-7.3$ & $2.6-8.5$ & {$[24]$} \\
\hline Frontal, Parietal, Occipital & II & $\mathrm{U}$ & Flat & - & Cadaver & $3.74-6.64$ & $4.1-6.9$ & {$[25]$} \\
\hline $\begin{array}{l}\text { Frontal, Temporo-Parietal, } \\
\text { Zygoma, Maxilla, Mandible }\end{array}$ & $\mathrm{I}$ & $\mathrm{PC}$ & Flat circular & Padded, $14.3 \mathrm{~mm}$ radius & Cadaver & $1.08-3.82$ & $2.99-5.97$ & {$[26]$} \\
\hline Temporo-Parietal & I & $\mathrm{C}$ & Flat circular & $12.7 \mathrm{~mm}$ radius & Cadaver & 10.6 & 2.7 & {$[27]$} \\
\hline Temporo-Parietal & I & $\mathrm{C}$ & Flat rectangular & $50 \mathrm{~mm} \times 100 \mathrm{~mm}$ & Cadaver & 12 & 4.3 & [27] \\
\hline Nose & I & $\mathrm{C}$ & Flat circular & $14.3 \mathrm{~mm}$ radius & Cadaver & 3.2 & $1.58-3.16$ & [28] \\
\hline Nose & III & $\mathrm{U}$ & Edge & $12.5 \mathrm{~mm}$ & Cadaver & 32,64 & $2.8-7.1$ & [29] \\
\hline Chest & IV & $\mathrm{PC}$ & Flat circular & $\begin{array}{l}\text { Padded, } 15.24 \mathrm{~cm} \text { diameter, } \\
161.29-193.55 \mathrm{~cm}^{2} \text { surface }\end{array}$ & Cadaver & $14.03-19.55$ & $4.52-10.06$ & {$[30],[31]$} \\
\hline Chest & III & $\mathrm{U}$ & Flat circular & $\begin{array}{l}15.24 \mathrm{~cm} \text { diameter, } \\
1.28 \mathrm{~cm} \text { edge radius }\end{array}$ & Cadaver & $1.63-23.59$ & $6.26-14.31$ & {$[32]$} \\
\hline Chest & III & $\mathrm{PC}$ & Flat circular & $\begin{array}{l}15.24 \mathrm{~cm} \text { diameter, } \\
1.28 \mathrm{~cm} \text { edge radius }\end{array}$ & Cadaver & 9.98 & $5.36-6.26$ & {$[33]$} \\
\hline Chest & IV & $\mathrm{U}$ & Flat circular & $\begin{array}{l}\text { Padded, } 15.24 \mathrm{~cm} \text { diameter, } \\
1.28 \mathrm{~cm} \text { edge radius }\end{array}$ & Volunteer & 10.01 & $2.40-4.60$ & {$[34]$} \\
\hline Chest & III & $\mathrm{U}, \mathrm{C}$ & Flat circular & Rigid/padded, $6.45 \mathrm{~cm}^{2}$ surface & Cadaver & $1.51,10.01$ & $4.02-10.01$ & {$[34]$} \\
\hline Chest & III & $\mathrm{U}, \mathrm{C}$ & Flat circular & $\begin{array}{l}15.24 \mathrm{~cm} \text { diameter } \\
1.28 \mathrm{~cm} \text { edge radius }\end{array}$ & Cadaver & $\begin{array}{l}1.59 \\
23.04\end{array}$ & $4.34-13.23$ & [35] \\
\hline Chest & III & $\mathrm{U}, \mathrm{PC}$ & Flat circular & $\begin{array}{l}15.24 \mathrm{~cm} \text { diameter } \\
1.28 \mathrm{~cm} \text { edge radius }\end{array}$ & $\begin{array}{l}\text { Porcine } \\
\text { (anesthetized) }\end{array}$ & 21.00 & $3.00-12.20$ & {$[36]$} \\
\hline Chest & II & $\mathrm{PC}$ & Flat circular & $\begin{array}{l}15.00 \mathrm{~cm} \text { diameter } \\
1.27 \mathrm{~cm} \text { edge radius }\end{array}$ & $\begin{array}{l}\text { Swine } \\
\text { (anesthetized) }\end{array}$ & $\begin{array}{l}4.90,10.40,21.00 \\
21.00\end{array}$ & $8.10-31.60$ & [37], [38] \\
\hline Chest & IV & $\mathrm{C}$ & Flat circular & - & Cadaver & $11.05-26.19$ & $6.44-16.61$ & [39] \\
\hline Chest & III & $\mathrm{U}, \mathrm{C}$ & Sphere & $12.0 \mathrm{~cm}$ radius & HIII Dummy & $4,67,1980$ & $0.20-4.20$ & [22] \\
\hline Chest & I & PC & Sphere & $12.5 \mathrm{~mm}$ radius & Volunteer & $3.68-3.79$ & $0.19-1.31$ & {$[12]$} \\
\hline & & & Edge & $0.2 \mathrm{~mm}$ edge radius, $20.0 \mathrm{~cm}$ length & & $4.28-3.57$ & $0.19-0.84$ & \\
\hline
\end{tabular}
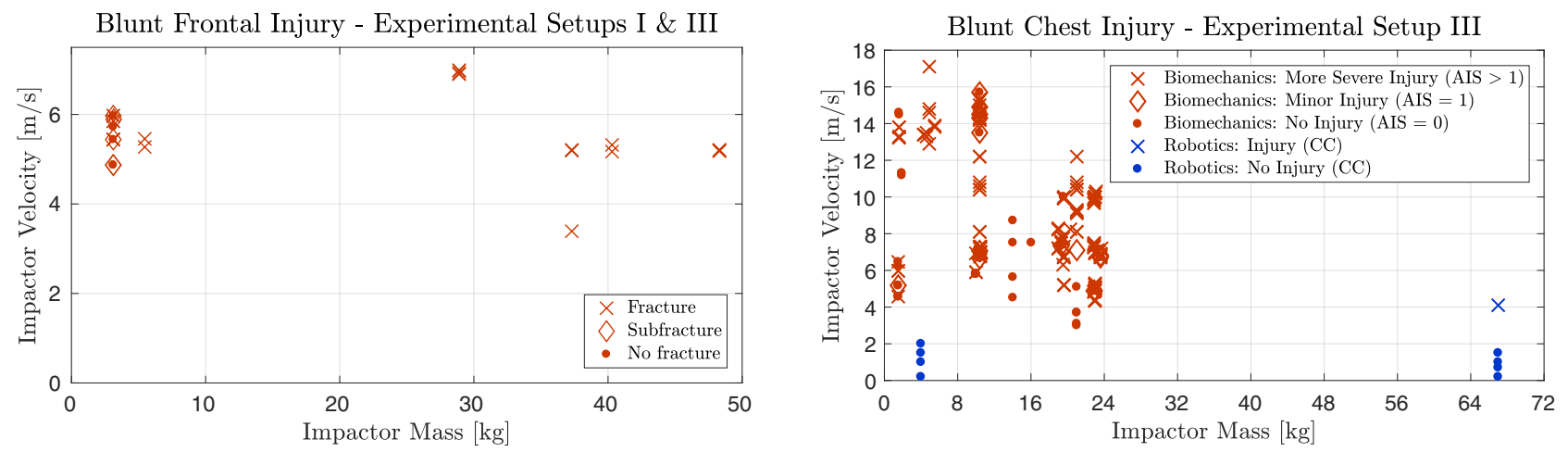

Fig. 3. Summary of relation between mass, velocity, and injury for selected data on the frontal bone (left, [20], [24], [26]) and chest (right, [22], [32]-[38]).

1) discretize the workspace and determine all reachable poses of a robot, in other words, its reachability map,

2) for each reachable pose, determine the set of reachable null space configurations if the robot is redundant,

3) generate a grid of Cartesian directions, and

4) calculate the Cartesian reflected mass and maximum velocity for each feasible pose, null space position, and Cartesian direction.

The overall approach is illustrated in Fig. 4. In the following, we explain the fours steps in more detail. Furthermore, we comment on the influence of an end-effector/load on the calculated mass/velocity range.

\section{A. Discretize Workspace \& Determine Reachable Workspace (Step 1)}

We seek to determine a robot's so-called versatile workspace, i.e., all (discretized) reachable combinations of
Cartesian position $\boldsymbol{t} \in \mathbb{R}^{3}$ and orientation $\boldsymbol{R} \in S O(3)$, which is defined as [41]

$$
\mathcal{W}_{v}=\left\{(\boldsymbol{t}, \boldsymbol{R}) \mid \exists \boldsymbol{q} \in \mathcal{Q}: g_{f k}(\boldsymbol{q})=\left[\begin{array}{cc}
\boldsymbol{R} & \boldsymbol{t} \\
\mathbf{0} & 1
\end{array}\right]\right\} \subset S E(3)
$$

Here, $\mathcal{Q}$ is the robot configuration space and $g_{f k}$ is the forward kinematics. Many algorithms exist to determine the versatile workspace. In algorithm [41], the workspace is discretized into an evenly-spaced, orthogonal position grid with desired granularity. We denote the cardinality of the set of possible positions $\mathcal{T}=\left\{\boldsymbol{t}_{1}, \ldots, \boldsymbol{t}_{n_{t}}\right\} \subset \mathbb{R}^{3}$ as $n_{t}$. For each position, a certain number $n_{r}$ of discretized end-effector orientations $\mathcal{R}=\left\{\boldsymbol{R}_{1}, \ldots, \boldsymbol{R}_{n_{r}}\right\} \subset S O(3)$ is defined. Forward and/or inverse kinematics can then be utilized to obtain the reachable poses and their associated joint position. 


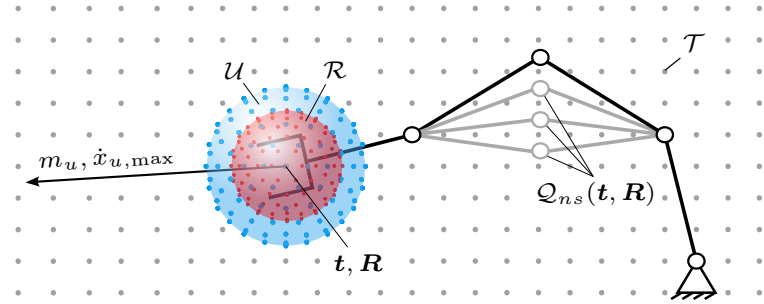

Fig. 4. Determining the global robot dynamic properties. The evenly-spaced Cartesian position grid is denoted $\mathcal{T}$, the current end-effector position and orientation $\boldsymbol{t}$ and $\boldsymbol{R}$, and the set of possible null space positions associated to this pose $\mathcal{Q}_{n s}(\boldsymbol{t}, \boldsymbol{R})$. The set of discretized end-effector orientations (red sphere) for which the reachability is analyzed (for each position) is denoted $\mathcal{R}$, the set of Cartesian unit directions (blue sphere) for evaluating the reflected robot mass and velocity $\mathcal{U}$. The current reflected mass and velocity in direction $\boldsymbol{u}$ are referred to as $m_{u}$ and $\dot{x}_{u, \max }$, respectively.

\section{B. Determine Null Space Configurations for Redundant Robots (Step 2)}

The reachability map usually provides one joint configuration for a desired Cartesian pose. If the robot is redundant, then additionally a desired number $n_{q_{n s}}$ of possible null space positions with their according mass and velocity can be calculated systematically ${ }^{3}$. For each Cartesian position $t \in \mathcal{T}$ and orientation $\boldsymbol{R} \in \mathcal{R}$ we denote the set of discretized, feasible null space configurations as $\mathcal{Q}_{n s}(\boldsymbol{t}, \boldsymbol{R})$.

In this paper, we analyze the non-redundant six-DOF PUMA 560 and the seven-DOF $L W R I V+$ in a static six-DOF position/orientation task. For this task, robots like the $L W R$ have one redundant degree of freedom if the configuration is nonsingular. The possible null space positions associated with a certain pose can be determined by successively integrating the one-dimensional kernel of the Jacobian matrix. Details on the integration procedure for the $L W R$ can be found in [42]. An analysis of the self-motion manifold of robots with more DOF can be found in [43], [44].

\section{Generate Grid of Cartesian Directions (Step 3)}

As the end-effector can move in every Cartesian direction (except in singular configurations), we want to determine the reflected mass and maximum velocity associated to each pose for a discretized number $n_{u}$ of Cartesian unit directions. For this, we generate a uniform grid on the surface of the unit sphere $\mathcal{S}^{2}=\left\{\boldsymbol{x} \in \mathbb{R}^{3}:\|\boldsymbol{x}\|=1\right\}$, where the set of distributed points is defined as $\mathcal{U}=\left\{\boldsymbol{u}_{1}, \ldots, \boldsymbol{u}_{n_{u}}\right\} \subset \mathcal{S}^{2}$.

If the joint position and velocity constraints are symmetric, then we only need to consider half of the sphere because the reflected mass $m_{u}$ and the magnitude of $\dot{x}_{u}$ are the same in directions $\boldsymbol{u}$ and $-\boldsymbol{u}$, respectively, cf. Fig. 2. The robot reflected mass and velocity for the other half of the sphere can be assigned by making use of this symmetry.

\section{Calculate Reflected Mass \& Maximum Velocity (Step 4)}

For all reachable poses, for all possible null space positions, and for all Cartesian directions, i. e., for at most $n_{t o t} \leq n_{t} \times$ $n_{r} \times n_{q_{n s}} \times n_{u}$ configurations, we finally evaluate the reflected mass and maximum Cartesian velocity at the robot flange, respectively the tool center point (TCP).

\footnotetext{
${ }^{3}$ Also non-redundant robots may have several possible joint configurations for a desired end-effector pose. However, for sake of brevity we omit a thorough analysis of such configurations in this work.
}

The reflected mass can be calculated with the equations provided in Fig. 2. In terms of speed, we want to evaluate the robot maximum possible velocity under consideration of the given constraints. For this, we formulate a static optimization problem which is described in the following.

Let us decompose the Cartesian velocity as $\dot{\boldsymbol{x}}=\left[\boldsymbol{\nu}^{\top}, \boldsymbol{\omega}^{\top}\right]^{\top}$, where $\boldsymbol{\nu} \in \mathbb{R}^{3}$ is the translational and $\boldsymbol{\omega} \in \mathbb{R}^{3}$ the angular velocity, respectively. The former shall satisfy $\boldsymbol{\nu}=\dot{x}_{u} \boldsymbol{u}$, where $\dot{x}_{u} \in \mathbb{R}$ is the magnitude of the velocity in direction $\boldsymbol{u}$. The cost function for our problem is

$$
J_{\mathrm{opt}}=\dot{x}_{u} \rightarrow \max
$$

In terms of angular velocity, we set $\boldsymbol{\omega}=\mathbf{0}_{3 \times 1}$ to purely move with a translational velocity ${ }^{4}$. The optimization is thus subject to the equality constraint

$$
\boldsymbol{J}(\boldsymbol{q}) \dot{\boldsymbol{q}}-\left[\begin{array}{c}
\dot{x}_{u} \boldsymbol{u} \\
\mathbf{0}_{3 \times 1}
\end{array}\right]=\mathbf{0}_{6 \times 1} .
$$

The joint velocity limits define the inequality constraints

$$
-\dot{\boldsymbol{q}}_{\max } \leq \dot{\boldsymbol{q}} \leq \dot{\boldsymbol{q}}_{\max }
$$

This optimization problem can be brought into standard form $\boldsymbol{A}_{\mathrm{eq}} \boldsymbol{x}_{\mathrm{opt}}=\boldsymbol{b}_{\mathrm{eq}}$ for (3) and $\boldsymbol{A} \boldsymbol{x}_{\mathrm{opt}} \leq \boldsymbol{b}$ for (4) by defining the state $\boldsymbol{x}_{\mathrm{opt}}=\left[\dot{x}_{u}, \dot{\boldsymbol{q}}^{\top}\right]^{\top}$ and solved efficiently via linear programming. Finally, we obtain the maximum velocity $\dot{x}_{u, \max }$ in direction $\boldsymbol{u}$. Due to the limited joint torque dynamics, $\dot{x}_{u, \max }$ is not always practically feasible. The found solution is therefore a conservative estimate.

Note again that the full calculation of the global dynamic properties needs to be done only once and significant computation time is therefore acceptable. However, once the data was generated, it can be accessed and processed efficiently.

\section{E. Influence of End-Effector/Payload on Reflected Mass and Maximum Velocity}

In this paper, we calculate the robot mass/velocity range for the robot flange or TCP. When attaching an endeffector/payload (constant inertia tensor around its center of gravity) to the system, then its mass and inertia influence the robot kinetic energy, and its geometry may influence the maximum Cartesian velocity. Ideally, one would determine the dynamic properties for all reachable poses only once and then shift/transform the mass/velocity ranges according to the specific tool parameters with only little computational effort.

When expressed relative to the operational point, the overall kinetic energy matrix is given by the addition of the robot's and the end-effector's kinetic energy matrices [45]. The reflected mass at the load in direction $\boldsymbol{u}$ increases with the specific load mass and inertia. If an angular velocity is present at the flange, then the tool geometry may have an influence on operational speed and the optimization procedure will provide a different solution for each location on the tool. A full analysis on this topic, however, goes significantly beyond the scope of this paper and is subject to future work.

\footnotetext{
${ }^{4}$ It is also possible to drop the constraint on angular velocity and allow for an arbitrary value or impose another reasonable constraint. To keep the discussion clear and make the motion intuitively interpretable, we only consider translational motions in this paper.
} 

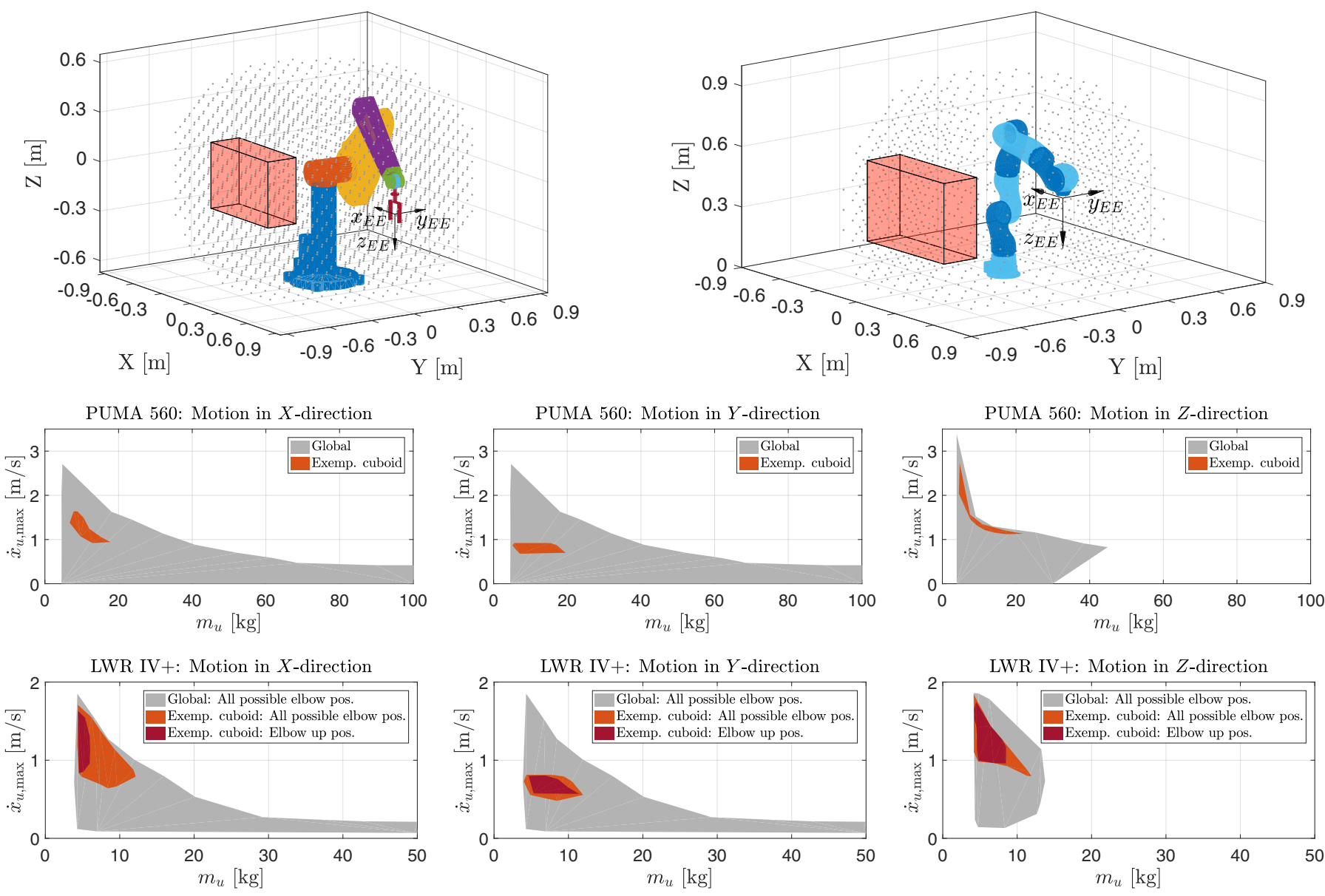

Fig. 5. Map representation of the PUMA 560 and the $L W R I V+$ for motions in Cartesian $X$-, $Y$-, and $Z$-direction. In the upper row, the feasible Cartesian positions for the considered end-effector orientation are illustrated as dots, an exemplary subset of the workspace, a cuboid with $60 \times 20 \times 40 \mathrm{~cm}$, is colored red. In the middle row, the mass/velocity representation of the entire PUMA 560 and of the exemplary cuboid are depicted. For the $L W R I V+$ results in the lower row, we also differentiate between the standard elbow-up configuration and all possible null space configurations for the exemplary cuboid.

\section{F. Results for PUMA 560 and LWR IV+}

Now, we determine the safety map representation of the sixDOF PUMA 560 and the seven-DOF $L W R I V+$. The results are illustrated in Fig. 5. For generating the Cartesian position grid, we select a $5 \mathrm{~cm}$ uniform distance between the positions. For sake of clarity, we only consider one end-effector orientation. For both robots, the end-effector axes $\boldsymbol{x}_{E E}, \boldsymbol{y}_{E E}$, and $\boldsymbol{z}_{E E}$ are aligned with the Cartesian axes $\boldsymbol{x}_{0}, \boldsymbol{y}_{0}$, and $\boldsymbol{z}_{0}$ as follows: $\boldsymbol{x}_{E E}=-\boldsymbol{x}_{0}, \boldsymbol{y}_{E E}=\boldsymbol{y}_{0}, \boldsymbol{z}_{E E}=-\boldsymbol{z}_{0}$. We want to analyze the reflected mass in the principal motion directions, i.e., we choose $\boldsymbol{u}=\boldsymbol{x}_{0}, \boldsymbol{u}=\boldsymbol{y}_{0}$, and $\boldsymbol{u}=\boldsymbol{z}_{0}$. In addition to the global mass/velocity range, we analyze the dynamic properties for a typical workspace area of $60 \times 20 \times 40 \mathrm{~cm}$ size, which is chosen to be the same for both robots.

For the PUMA 560, we use the inverse kinematics algorithm [46] and select an elbow-up and so-called "lefty" configuration as the preferred configuration. For the considered problem, we identify 19837 feasible poses. In Fig. 5 (middle row), we illustrate the accumulated mass/velocity range of the robot for translational motions in Cartesian $X-, Y$-, and $Z$-direction. For the LWR's inverse kinematics algorithm [47], we select an elbow-up configuration as the standard configuration. We find 9138 positions and determine 15 null space configurations for each non-singular configuration. In Fig. 5 (lower row) we show the global $X, Y$, and $Z$ mass/velocity range of the robot including null space motions. Please note that we illustrate the maximum possible velocity in Fig. 5. Of course, the robots can always travel with lower speed, meaning the area below the illustrated mass/velocity ranges is feasible as well.

The boundary of the robots' safety map representation is mainly defined by the dynamic properties in singular or near-singular configurations. When the robot approaches the workspace boundary, then the reflected mass in direction of the robot structure becomes very high while the maximum velocity becomes very low. If the robot is outstretched but does not point direction of a Cartesian axis, then the reflected mass in $X$-, $Y$-, or $Z$-direction is in a "normal" range but the maximum velocity is still very low due to the robot configuration being singular. The maximum possible velocity can be reached either in singular or non-singular configurations. The results for the exemplary cube indicate the reflected mass and maximum velocity ranges that can be expected in a typical workspace area. These results will be used again in the use case considered in the next section.

\section{Vi. Application of Safety Map to Safety ASSESSMENT AND Robot/TASK DESIGN}

In the following, we describe how the safety map can be integrated into the robot and task design workflow as a safety evaluation tool. If one is interested in the global 


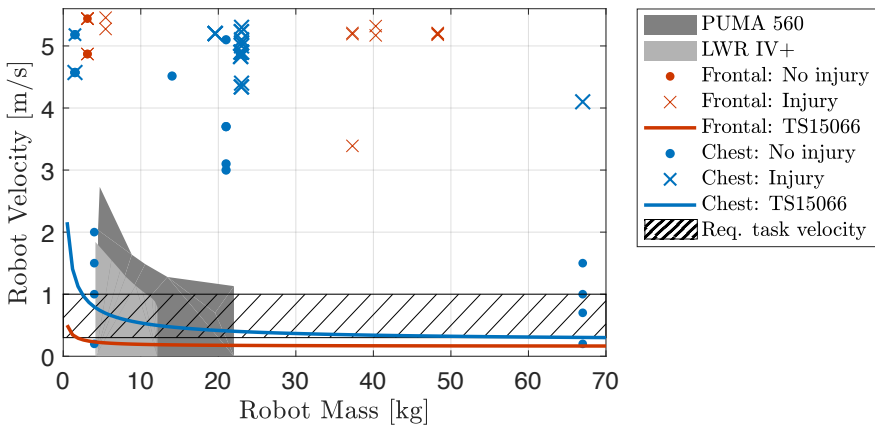

Fig. 6. Safety map for pick and place use case. The mass/velocity range of the PUMA 560 and $L W R I V+$ for the considered workspace area are illustrated in dark/light gray, head and chest biomechanics injury data in red and blue, and the required task velocity in black color.

dynamic properties of the robot without having a specific application at hand, then the safety map can be utilized to analyze, e.g., whether a) the robot is capable of producing a certain type of injury, b) where the most dangerous areas in the reachable workspace are located, or c) how safety properties compare with other performance indices such as manipulability/dexterity in certain workspace areas.

For assessing certain applications with a given robot in terms of safety, following steps have to be carried out to obtain the safety map representation for the considered task:

1) Extract task-dependent mass/velocity data (workspace area or trajectory) from global robot dynamic properties,

2) Assign contact primitives with their parameters to points of interest on the robot structure (usually the endeffector),

3) Identify collision scenarios (constrained/unconstrained) and human body parts that may be hit during collisions by analyzing the shared workspace, and

4) Select corresponding injury data and relevant thresholds from the current standards.

If the robot and injury data intersect in the safety map, then we can conclude that a collision of this dynamic properties will likely result in an injury when the robot always travels at maximum speed. In this situation, we can determine the (configuration dependent) maximum safe velocity that can be applied. Additionally, we can analyze whether certain performance requirements (cycle time) in terms of a desired velocity range can be met by the selected robot. If the desired velocity range, robot and injury data intersect, then one must take countermeasures in either control/planning or mechanical/task design. If the performance requirements cannot be met by control, then one should analyze whether it is possible to modify the robot's critical geometries and/or add padding. If safety still cannot be improved via mechanical modifications, then task/workspace design changes must be made or another robot must be selected for the task.

Please note that the framework allows for different degrees of granularity in the safety assessment. In terms of the robot's safety map representation, one may either use a) the global dynamic properties, b) a task-dependent subset, or c) extract the data associated with a certain trajectory if the trajectory has been planned already. Furthermore, the calculation of the robot dynamic properties can be done with either fine or coarse grids $\mathcal{T}, \mathcal{Q}_{n s}, \mathcal{R}$, and $\mathcal{U}$, which makes short iterations in robot design and successive safety evaluation possible.

\section{A. Pick \& Place Task}

Next, we apply the safety map framework to a practical pick and place task. Both the PUMA 560 and LWR IV+ shall perform translational motions in Cartesian $X-, Y$-, or $Z$-direction in the exemplary cuboid depicted in Fig. 5 (upper row, same workspace for both robots). The task requires the robot velocity to be in the range $0.3-1 \mathrm{~m} / \mathrm{s}$. In Fig. 6, we illustrate the combined robot mass/velocity ranges for both robots. The robot data is compared to injury data for blunt, unconstrained impacts against the human frontal bone (cf. Fig. 3 (left)), blunt chest injury data (cf. Fig. 3 (right)), and the current ISO/TS 15066 thresholds. Please note that the TS 15066 relates force/pressure limits $\left(1 \mathrm{~cm}^{2}\right.$ contact area) to thresholds in the mass/velocity plane via a simplified contact model. The thresholds were not derived from experiments which aim at finding a data-driven relation between collision input parameters and human injury. Furthermore, it is currently not entirely clear where part of the reported force/pressure limits originate from.

For the considered task, the PUMA 560 can reach higher velocities and typically it has a higher reflected mass than the $L W R I V+$. In Fig. 6 the ranges of feasible mass and velocity pairs for both robots remain well below the critical values of the biomechanics data ${ }^{5}$. The pure fact that the considered types of injury are unlikely to occur for these robot mass/velocity ranges was already shown in [22]. However, to the best of the authors' knowledge, the global, systematic analysis and comparison of robot mass and velocity range and the relation to real biomechanics injury data has not been done until now.

While no severe injury is to be expected, the TS 15066 threshold may be violated by both robots. The desired task velocity indicates that, e. g., speed limitation via planning/control can account for both chest collision safety and performance requirements. For the considered task and injury data, head collision safety can not be guaranteed when moving with the specified velocities, which means that further modifications in mechanical or task design are necessary.

\section{CONCLUSION}

In this paper, we proposed the concept of a safety map, a global map that serves as a common unified representation for injury biomechanics data and robot collision behavior. The safety map is a novel tool for robot developers that can be utilized for injury analysis and safer robot design already at an early concept phase of the design and development process. The mass and velocity range of the entire robot workspace, or task-dependent subspaces, can be quantitatively compared to any available injury data for different contact primitives, collision cases, and human body parts. This gives the designer clear information which kind of injury is most likely to occur during operation, thus guiding not only the hardware design process, but also giving valuable information to safe interaction control and motion planning algorithm development. In fact, the safety map can also be directly employed as a cost map for robot safety-oriented motion planning or as a global cost function for optimal control. In this paper, we validated our approach using the dynamics of the six-DOF PUMA 560 and the seven-DOF KUKA Lightweight Robot IV+. We determined the safety map representation for both robots and related them to

\footnotetext{
${ }^{5}$ Please note that we illustrate raw biomechanics data and no classification in terms of injury/no injury.
} 
biomechanics injury data, that was classified, validated, and processed during a thorough biomechanics literature survey. To the best of the authors' knowledge, the presented framework is the first global dynamic and exact safety analysis tool for robot manipulators, which may lead to significant changes in the way human-friendly robots are designed in the future.

\section{REFERENCES}

[1] A. Bicchi and G. Tonietti, "Fast and soft arm tactics: Dealing with the safety-performance trade-off in robot arms design and control," IEEE Robotics and Automation Magazine, vol. 11, pp. 22-33, 2004.

[2] J.-J. Park, H.-S. Kim, and J.-B. Song, "Safe robot arm with safe joint mechanism using nonlinear spring system for collision safety," in IEEE International Conference on Robotics and Automation (ICRA2009). IEEE, 2009, pp. 3371-3376.

[3] J.-J. Park, S. Haddadin, J. Song, and A. Albu-Schäffer, "Designing optimally safe robot surface properties for minimizing the stress characteristics of human-robot collisions," in IEEE Int. Conf. on Robotics and Automation (ICRA2011), Shanghai,China, 2011, pp. 5413-5420.

[4] S. Haddadin, A. Albu-Schäffer, O. Eiberger, and G. Hirzinger, "New insights concerning intrinsic joint elasticity for safety," in IEEE/RSJ Int. Conf. on Intelligent Robots and Systems (IROS2010), Taipeh, Taiwan, 2010, pp. 2181-2187.

[5] K. T. Ulrich, T. Tuttle, J. P. Donoghue, and W. T. Townsend, "Intrinsically safer robots," Barrett Technology, Inc., Project Report, 1995.

[6] K. Ikuta, H. Ishii, and M. Nokata, "Safety evaluation method of design and control for human-care robots," International Journal of Robotics Research, vol. 22, no. 5, pp. 281-298, 2003.

[7] D. Kulic and E. Croft, "Pre-collision strategies for human robot interaction," Autonomous Robots, vol. 22, no. 2, pp. 149-164, 2007.

[8] B. Lacevic, P. Rocco, and A. M. Zanchettin, "Safety assessment and control of robotic manipulators using danger field," IEEE Transactions on Robotics, vol. 29, no. 5, pp. 1257-1270, 2013.

[9] T. S. Tadele, T. J. de Vries, and S. Stramigioli, "Combining energy and power based safety metrics in controller design for domestic robots," in IEEE International Conference on Robotics and Automation (ICRA2014). IEEE, 2014, pp. 1209-1214.

[10] R. Rossi, M. P. Polverini, A. M. Zanchettin, and P. Rocco, "A precollision control strategy for human-robot interaction based on dissipated energy in potential inelastic impacts," in IEEE/RSJ International Conference on Intelligent Robots and Systems (IROS2015). IEEE, 2015, pp. 26-31.

[11] S. Haddadin, S. Haddadin, A. Khoury, T. Rokahr, S. Parusel, R. Burgkart, A. Bicchi, and A. Albu-Schäffer, "On making robots understand safety: Embedding injury knowledge into control," The International Journal of Robotics Research, vol. 31, no. 13, pp. 15781602,2012

[12] S. Haddadin and E. Croft, "Physical human-robot interaction," in Handbook of Robotics, 2nd ed., B. Siciliano and O. Khatib, Eds. Springer, 2016.

[13] V. Hodgson, J. Brinn, L. Thomas, and S. Greenberg, "Fracture behavior of the skull frontal bone against cylindrical surfaces," in 14th Stapp Car Crash Conference. SAE International, 1970, pp. 341-355.

[14] D. Allsop, C. Warner, M. Wille, D. Schneider, and A. Nahum, "Facial impact response - a comparison of the Hybrid III dummy and human cadaver," in 32th Stapp Car Crash Conference. SAE International, 1988, pp. 781-797.

[15] O. Khatib, "Inertial properties in robotic manipulation: an object-level framework," The International Journal of Robotics Research, vol. 14, no. 1 , pp. $19-36,1995$.

[16] F. H. Netter, Atlas of human anatomy. Elsevier Health Sciences, 2014.

[17] R. Chandler, C. E. Clauser, J. T. McConville, H. Reynolds, and J. W. Young, "Investigation of inertial properties of the human body," DTIC Document, Tech. Rep., 1975.

[18] M. Mungiole and P. E. Martin, "Estimating segment inertial properties: comparison of magnetic resonance imaging with existing methods," Journal of Biomechanics, vol. 23, no. 10, pp. 1039-1046, 1990.

[19] S. Haddadin, A. Albu-Schäffer, and G. Hirzinger, "Requirements for safe robots: Measurements, analysis \& new insights," Int. J. of Robotics Research, vol. 28, no. 11-12, pp. 1507-1527, 2009.

[20] H. Delye, P. Verschueren, B. Depreitere, I. Verpoest, D. Berckmans, J. Vander Sloten, G. Van Der Perre, and J. Goffin, "Biomechanics of frontal skull fracture," Journal of Neurotrauma, vol. 24, no. 10, pp. $1576-1586,2007$

[21] K. Ono, A. Kikuchi, M. Nakamura, H. Kobayashi, and N. Nakamura, "Human head tolerance to sagittal impact reliable estimation deduced from experimental head injury using subhuman primates and human cadaver skulls," in 24th Stapp Car Crash Conference. SAE International, 1980.
[22] S. Haddadin, A. Albu-Schäffer, M. Frommberger, J. Rossmann, and G. Hirzinger, "The "DLR Crash Report": Towards a standard crashtesting protocol for robot safety - part I: Results," in IEEE Int. Conf. on Robotics and Automation (ICRA2008), Kobe, Japan, 2009, pp. 272-279.

[23] V. Hodgson and L. Thomas, "Breaking strength of the human skull versus impact surface curvature," Wayne State University, Detroit, Final Report DOT HS-801 002, 1973.

[24] V. Hodgson, "Tolerance of the facial bones to impact," American Journal of Anatomy, vol. 120, no. 1, pp. 113-122, 1967.

[25] E. Gurdjian, J. E. Webster, and H. R. Lissner, "Studies on skull fracture with particular reference to engineering factors," The American Journal of Surgery, vol. 78, no. 5, pp. $736-742,1949$.

[26] D. Schneider and A. Nahum, "Impact studies of facial bones and skull," in 16th Stapp Car Crash Conference, 1972, pp. 186-204.

[27] D. Allsop, T. Perl, and C. Warner, "Force/deflection and fracture characteristics of the temporo-parietal region of the human head," SAE Transactions, pp. 2009-2018, 1991.

[28] J. Cormier, S. Manoogian, J. Bisplinghoff, S. Rowson, A. Santago, C. McNally, S. Duma, and J. Bolte Iv, "The tolerance of the nasal bone to blunt impact," in Annals of Advances in Automotive Medicine/Annual Scientific Conference, vol. 54, 2010, p. 3.

[29] G. W. Nyquist, J. M. Cavanaugh, S. J. Goldberg, and A. I. King, "Facial impact tolerance and response," in 30th Stapp Car Crash Conference, 1986, pp. 733-754.

[30] L. Patrick, C. Kroell, and H. Mertz, "Forces on the human body in simulated crashes," in 9th Stapp Car Crash Conference. SAE International, 1965, pp. 237-260.

[31] L. Patrick, H. Mertz, and C. Kroell, "Cadaver knee, chest, and head impact loads," in 11th Stapp Car Crash Conference. SAE International, 1967 , pp. $168-182$.

[32] C. Kroell, D. Schneider, and A. Nahum, "Impact tolerance and response of the human thorax," in 15th Stapp Car Crash Conference. SAE International, 1971, pp. 84-134.

[33] R. L. Stalnaker, J. H. McElhaney, V. L. Roberts, and M. L. Trollope, Human Torso Response to Blunt Trauma. Boston, MA: Springer US, 1973, pp. 181-199.

[34] A. M. Nahum, C. W. Gadd, D. C. Schneider, and C. Kroell, "Deflection of the human thorax under sternal impact," in International Automobile Safety Conference, 1970.

[35] C. Kroell, D. Scheider, and A. Nahum, "Impact tolerance and response of the human thorax II," in 18th Stapp Car Crash Conference. SAE International, 1974, pp. 383-457.

[36] D. Viano and C. Warner, "Thoracic impact response of live porcine subjects," in 20th Stapp Car Crash Conference. SAE International, 1976, pp. 441-474.

[37] C. K. Kroell, M. E. Pope, D. C. Viano, C. Y. Warner, and S. D. Allen, "Interrelationship of velocity and chest compression in blunt thoracic impact to swine," in 25th Stapp Car Crash Conference. SAE International, 1981

[38] C. K. Kroell, S. D. Allen, C. Y. Warner, and T. R. Perl, "Interrelationship of velocity and chest compression in blunt thoracic impact to swine II," in 30th Stapp Car Crash Conference. SAE International, 1986.

[39] S. M. Kuppa and R. H. Eppinger, "Development of an improved thoracic injury criterion," in 42nd Stapp Car Crash Conference. SAE International, 1998, pp. 139-154.

[40] S. Haddadin, A. Albu-Schäffer, M. Frommberger, J. Rossmann, and G. Hirzinger, "The "DLR Crash Report": Towards a standard crashtesting protocol for robot safety - part II: Discussions," IEEE Int. Conf. on Robotics and Automation (ICRA2008), Kobe, Japan, pp. 280-287, 2009.

[41] F. Zacharias, Knowledge representations for planning manipulation tasks. Springer Science \& Business Media, 2012, vol. 16.

[42] N. Mansfeld, B. Djellab, J. Raldúa Veuthey, F. Beck, C. Ott, and S. Haddadin, "Improving the performance of biomechanically safe velocity control for redundant robots through reflected mass minimization," in International Conference on IEEE/RSJ Intelligent Robots and Systems (IROS2017), Vancouver, Canada, 2017

[43] J. W. Burdick, "On the inverse kinematics of redundant manipulators: Characterization of the self-motion manifolds," in IEEE International Conference on Robotics and Automation (ICRA1989). IEEE, 1989, pp. 264-270.

[44] C. L. Luck and S. Lee, "Redundant manipulator self-motion topology under joint limits with an 8-dof case study," in IEEE/RSJ International Conference on Intelligent Robots and Systems (IROS1993), vol. 2. IEEE, 1993, pp. 848-855.

[45] O. Khatib, "Object manipulation in a multi-effector robot system," in ISRR, R. Bolles and B. Roth, Eds. Santa Cruz, CA, USA: The MIT Press, Cambridge, MA, USA, 5 1988, pp. 137-144.

[46] P. Corke, Robotics, vision and control: fundamental algorithms in MATLAB. Springer, 2011, vol. 73.

[47] R. Konietschke, Planning of Workplaces with Multiple Kinematically Redundant Robots. Technische Universität München, 2008. 